\title{
Impact of Interference Correlation on the Decoding Error Statistics
}

\author{
Fernando Rosas ${ }^{1,2}$, Konstantinos Manolakis ${ }^{3}$, Christian Oberli ${ }^{4}$, M. Mahdi Azari ${ }^{5}$, \\ Marian Verhelst ${ }^{5}$ and Sofie Pollin ${ }^{5}$ \\ ${ }^{1}$ Centre for Complexity Science and Department of Mathematics, Imperial College London, London, UK \\ 2 Department of Electrical and Electronic Engineering, Imperial College London, London, UK \\ ${ }^{3}$ Huawei Technologies, European Research Center, Munich, Germany \\ 4 Pontificia Universidad Católica de Chile, Departmento de Ingeniería Eléctrica, Santiago, Chile \\ ${ }^{5}$ Departement Elektrotechniek, KU Leuven, Leuven, Belgium
}

\begin{abstract}
Scenarios where interference is correlated with useful signals are often met in communications systems. Interestingly, even though uncorrelated interference mitigation has been extensively studied by the literature, there is still lack of insight into how correlation can affects information transfer processes. In this paper we formally address this issue and show how a positive correlation between the interference and useful signal power introduces a non-intuitive increase in the diversity gain, improving the error statistics with respect to the uncorrelated case. In contrast, the diversity gain decreases under negative correlation, degrading the system performance. These findings are confirmed by simulations for scenarios of practical relevance.
\end{abstract}

\section{INTRODUCTION}

Interference constitutes one of the main limitations for the performance of wireless communication networks, and its mitigation has been a persistent aim for decades. Moreover, it is expected that interference will become a main bottleneck for future dense wireless networks [1]. As a step before designing efficient compensation techniques, a deep understanding of the structure and effects of interference is needed.

Interference over communication links can be classified in two broad categories: interference that is statistically independent of the useful data signal and interference that is somehow correlated with it. The former category has been extensively studied in the literature and advanced tools are available for analyzing it (e.g. [2], [3]). In contrast, the latter category has only been partially investigated, even though examples of correlated interference abound in modern wireless communication systems.

The literature that studies scenarios with correlated interference focuses on specific transmission schemes. For example, the outage probability and the bit error rate (BER) of code division multiple access (CDMA) systems under correlated interference was studied in [4], where it was shown that a positive correlation improves the error statistics. The error statistics of a multiple-input multiple-output (MIMO) receiver decoding BPSK transmissions were analyzed in [5] and [6] for correlated angular domains of the interference and the signal of interest. Also, a study of systems where the interference undergoes the same fading coefficient than the desired signal are studied is presented in [7].
Even though the literature acknowledges the fact that correlations between interference and desired signal affects the system performance, no deep understanding on the rationale behind this effect is available yet. In this work we address this issue by analysing how a statistical dependency between the interference and the useful signal power - e.g. through the channel coefficient - affects the symbol error rate (SER). It is shown how a positive correlation increases the diversity gain of the system with respect to an equivalent uncorrelated case, whereas the diversity gain decreases when a negative correlation is present. To illustrate this ideas we present case studies of high practical interest.

A key element of our analysis is the conditional signal-tointerference-plus-noise ratio (SINR). One of our goals is to show that the conditional SINR is more accurate than other popular metrics for describing the performance of a channel with correlated interference. The concept of conditional signalto-interference ratio (SIR) was first introduced in [8], and later was used in [9] for building a framework that uses conditional probabilities for computing error rates in scenarios not affected by interference.

The rest of this work is organized as follows. Section II introduces the system model, and specializes it for two scenarios. Then, Section III presents an analysis of the role of the correlation sign. Simulation results are provided in Section IV and conclusions are summarized in Section V.

\section{System Model AND INTERfEREnCE ANALYSiS}

This section first presents our general system model (Section II-A), which is then further specified for cases of practical interest (Section II-B). Note that the main results of Section II-B1 are an original contributions of this work, while Section II-B2 closely follows [10], [11].

\section{A. System Model}

Consider a linear channel given by

$$
y=h x+I+n .
$$

where $y$ is the received signal, $x$ is the information-bearing complex symbol, $h$ is the complex channel coefficient, $I$ is an 
additive complex-valued interference term and $n$ is additive white Gaussian noise (AWGN). It is assumed that all the variables have zero mean and that $x$ and $I$ are statistically independent. Let us denote $E_{\mathrm{s}}=\mathbb{E}\left\{|x|^{2}\right\}, \sigma_{\mathrm{I}}^{2}=\mathbb{E}\left\{|I|^{2}\right\}$ and $\sigma_{\mathrm{n}}^{2}=\mathbb{E}\left\{|n|^{2}\right\}$ the mean received power of data, interference and noise, respectively, with $\mathbb{E}\{\cdot\}$ the expectation operator. As a convention we assume that $\mathbb{E}\left\{|h|^{2}\right\}=1$.

Let us now consider the case where there exists correlation between the interference power $|I|^{2}$ and the channel coefficient $h$. Correlation implies that $\mathbb{P}\left\{|I|^{2}=x \mid h\right\} \neq \mathbb{P}\left\{|I|^{2}=x\right\}$, where $\mathbb{P}\{A \mid B\}$ denotes the conditional probability of the event $A$ given that the event $B$ happened. As a way of keeping track of potential interdependencies, let us define the conditional interference power as

$$
\kappa_{\mathrm{I}}(h):=\mathbb{E}\left\{|I|^{2} \mid h\right\} .
$$

Above, $\mathbb{E}\left\{|I|^{2} \mid h\right\}$ is the conditional expected value of $|I|^{2}$ for a given realization of $h$, which corresponds to the average value of $|I|^{2}$ computed over the distribution $\mathbb{P}\left\{|I|^{2} \mid h\right\}$ for a fixed $h$ and varying $|I|^{2}$.

In general, $\kappa_{\mathrm{I}}(h)$ is a function of $h$ and hence a random variable*. Please note that $\kappa_{\mathrm{I}}(h)$ is always well-defined, and that it becomes a mere constant if and only if $|I|^{2}$ and $h$ are statistically independent. Therefore, is clear that $\kappa_{\mathrm{I}}(h)$ is capable of keeping track of both linear and non-linear correlations, being more powerful than a mere Pearson's correlation coefficient. Also, it is interesting to note that

$$
\mathbb{E}\left\{\kappa_{\mathrm{I}}(h)\right\}=\mathbb{E}\left\{\mathbb{E}\left\{|I|^{2} \mid h\right\}\right\}=\mathbb{E}\left\{|I|^{2}\right\}=\sigma_{\mathrm{I}}^{2},
$$

where the second equality is a well-known property of conditional expectations. This means that $\kappa_{\mathrm{I}}(h)$ describes the fluctuations of the interference power around its mean value caused by different values of $h$.

For a number of cases of interest, $\kappa_{\mathrm{I}}(h)$ only depends on the channel power $|h|^{2}$. For these cases, we denote as positively correlated interference (resp. negatively correlated interference) the case in which $\kappa_{\mathrm{I}}(h)$ is an increasing (resp. decreasing) function of $|h|^{2}$.

\section{B. Model Specialization and Interference Analysis}

In the sequel, cases of positively and negatively correlated interference are studied.

1) ICI in OFDM: In the presence of inter-carrier interference (ICI), the signal received on an orthogonal frequency division multiplexing (OFDM) subcarrier $k$ can be modeled as [13], [14]

$$
y_{k}=\beta_{k, k} h_{k} x_{k}+I_{k}^{\mathrm{ICI}}+n_{k},
$$

where $\beta_{k, k}$ expresses the distortion of the transmitted signal over subcarrier $k^{\dagger}, h_{k}$ is the corresponding channel coefficient,

\footnotetext{
*A rigurous exposition of the properties of the conditional mean value can be found in [12, Vol. II, Ch. VIII]

${ }^{\dagger}$ Expressions for $\beta_{k, \nu}$ can be found in [13] and [14], where it is shown that for typical parameter values $\left|\beta_{k, k}\right|^{2} \approx 1$.
}

$n_{k}$ is the noise term and $I_{k}^{\mathrm{ICI}}$ is the ICI from the other subcarriers given by

$$
I_{k}^{\mathrm{ICI}}=\sum_{\substack{\nu=-N_{\mathrm{s}} / 2 \\ \nu \neq k}}^{N_{\mathrm{s}} / 2-1} \beta_{k, \nu} h_{\nu} x_{\nu} .
$$

By using (5) in (2), and considering the statistical independence between $\beta_{k, \nu}, h_{\nu}$ and $x_{\nu}$, the conditional interference power is found to be

$$
\kappa_{\mathrm{I}}\left(h_{k}\right)=E_{\mathrm{s}} \sum_{\substack{\nu=-\frac{N_{\mathrm{s}}}{2} \\ \nu \neq k}}^{\frac{N_{\mathrm{s}}}{2}-1} g_{k, \nu} \mathbb{E}\left\{\left|h_{\nu}\right|^{2} \mid h_{k}\right\},
$$

where $g_{k, \nu}:=\mathbb{E}\left\{\left|\beta_{k, \nu}\right|^{2}\right\}$.

Note that $\mathbb{E}\left\{\left|h_{\nu}\right|^{2} \mid h_{k}\right\}$ is a non-linear estimator of $\left|h_{\nu}\right|^{2}$ in terms of $h_{k}$ [15, Ch. 1.2]. Considering a linear MMSE estimator for $\left|h_{\nu}\right|^{2}$ given $\left|h_{k}\right|^{2}$, denoted as $\left|h_{\nu}\right|_{\text {MMSE }}^{2}$, one finds that $[15$, Ch. 3.1.]

$$
\left|h_{\nu}\right|_{\text {MMSE }}^{2}=1+\rho_{k, \nu}\left(\left|h_{k}\right|^{2}-1\right),
$$

where $\rho_{k, \nu}$ denotes the Pearson correlation coefficient between $\left|h_{k}\right|^{2}$ and $\left|h_{\nu}\right|^{2}$. Using $\left|h_{\nu}\right|_{\text {MMSE }}^{2}$ as an approximation for $\mathbb{E}\left\{\left|h_{\nu}\right|^{2} \mid h_{k}\right\}$ and applying it in (6), one obtains

$$
v=\sum_{\substack{\nu=-\frac{N_{\mathrm{s}}}{2} \\ \nu \neq k}}^{\kappa_{\mathrm{I}}\left(h_{k}\right) \approx\left(u+v\left|h_{k}\right|^{2}\right) E_{\mathrm{s}}, \text { with }} g_{k, \nu} \rho_{k, \nu} \quad \text { and } \quad u=\sum_{\substack{\nu=-\frac{N_{\mathrm{s}}}{2} \\ \nu \neq k}}^{\frac{N_{\mathrm{s}}}{2}-1} g_{k, \nu}\left(1-\rho_{k, \nu}\right) .
$$

It is known from [13] that $g_{k, \nu}$ is a decreasing function of the subcarrier distance $|k-\nu|$, and therefore $\kappa_{\mathrm{I}}\left(h_{k}\right)$ is mainly affected by the neighboring subcarriers. In fact, those are the channels with which the subcarrier is most strongly correlated, having $\rho_{k, \nu} \geq 0$. Hence in general $v \geq 0$, and therefore $\kappa_{\mathrm{I}}\left(h_{k}\right)$ as given by (8) grows with $\left|h_{k}\right|^{2}$, showing a positively correlated interference.

2) Channel Estimation Errors in impulse-radio ultrawideband (IR-UWB): Consider IR-UWB transmissions over a broadband channel of $L$ multipath components with complex amplitude $h_{l}$ and delay $\tau_{l}$. A IR-UWB RAKE receiver consist of a collection of correlators focused on specific signal paths. The output signal of each RAKE correlator is $r_{l}=h_{l} x+n_{l}$, where $x$ is the transmitted symbol and $n_{l}$ is an AWGN term with mean power $\mathbb{E}\left\{\left|n_{l}\right|^{2}\right\}=\sigma_{\mathrm{n}}^{2} / E_{\mathrm{s}}$ [11]. The output signals of the correlators is then combined using maximum ratio combining (MRC).

Following [11], we consider the case where where the path delay $\tau_{l}$ is perfectly known, while the amplitude is estimated with an additive error as $\hat{h}_{l}=h_{l}+\epsilon_{l}$. The estimation error $\epsilon_{l}$ is assumed to be Gaussian with zero mean and variance $\mathbb{E}\left\{\left|\epsilon_{l}\right|^{2}\right\}=\sigma_{\mathrm{n}}^{2} /\left(M_{\mathrm{p}} E_{\mathrm{s}}\right)$, where $M_{\mathrm{p}}$ is the number of pilot 
signals used for channel estimation [11]. Then, the signal at the output of the RAKE-MRC receiver is

$$
y=\frac{1}{\|\boldsymbol{h}\|} \sum_{l=1}^{L} \hat{h}_{l}^{*} r_{l}=\|\boldsymbol{h}\| x+I_{\mathrm{UWB}}+\sum_{l=1}^{L} \frac{h_{l}^{*}}{\|\boldsymbol{h}\|} n_{l},
$$

where $\|\boldsymbol{h}\|$ is the Euclidian norm of the vector $\boldsymbol{h}=$ $\left(h_{1}, \ldots, h_{L}\right)$. Above, the interference generated by the imperfect channel estimation is given by

$$
I_{\mathrm{UWB}}=\frac{1}{\|\boldsymbol{h}\|} \sum_{l=1}^{L} \epsilon_{l}^{*}\left(h_{l} x+n_{l}\right) .
$$

Path amplitudes are normally uncorrelated, as they are caused by independent multipath propagation. Hence, using (11) in (2), the conditional interference power can be found to be [11]

$$
\kappa_{\mathrm{I}}(\boldsymbol{h})=\frac{\sigma_{\mathrm{n}}^{2}}{M_{p} E_{\mathrm{s}}}\left(1+\frac{L \sigma_{\mathrm{n}}^{2}}{\|\boldsymbol{h}\|^{2} E_{\mathrm{s}}}\right) .
$$

As here $\kappa_{\mathrm{I}}(\boldsymbol{h})$ is a decreasing function of $\|\boldsymbol{h}\|^{2}$, this corresponds to a case of negatively correlated interference.

\section{SER ANALYSIS WITH INTERFERENCE CORRELATION}

This section presents an analysis of the effect of correlated interference over the mean SER. In the sequel, Section III-A derives an expression for the conditional SINR, and Section III-B uses this result for explaining the role of the correlation sign on the channel diversity.

\section{A. SER and the Conditional SINR}

A decoding error event over a link represented by (1) can be modeled as a Bernoulli variable as

$$
\mathcal{E}= \begin{cases}1 & \text { if a decoding error was made } \\ 0 & \text { otherwise }\end{cases}
$$

Using this definition, the mean SER, denoted as $\bar{P}_{\mathrm{s}}$, can be computed as the expected value of $\mathcal{E}$. Moreover, using nested conditional expectations, it can be seen that

$$
\bar{P}_{\mathrm{s}}=\mathbb{E}\{\mathcal{E}\}=\mathbb{E}\{\mathbb{E}\{\mathcal{E} \mid h\}\},
$$

where $\mathbb{E}\{\mathcal{E} \mid h\}$ is the average over the interference and noise for a given channel realization $h$, while the outer operator averages over the channel realizations.

In many scenarios, including the ones considered in Subsection II-B, the interference is generated by a large number of additive contributors and hence its statistics converge to a Gaussian distribution according to the central limit theorem ${ }^{\ddagger}$. Hence, for a given $h$, the term $I+n$ distributes as a circularly symmetric complex Gaussian random variable with zero mean and variance $\kappa_{\mathrm{I}}(h)+\sigma_{\mathrm{n}}^{2}$. Therefore, the term $\mathbb{E}\{\mathcal{E} \mid h\}$ corresponds to the error rate of an AWGN channel with thermal noise term equal to $I+n$. In particular, using (14) and the well-known expressions for the SER of $M$-QAM modulations [16], the mean SER of BPSK and M-QAM transmissions is

${ }^{\ddagger}$ For the precise conditions required by the central limit theorem, c.f. [12, Vol. I, Sec. 23]

$$
\bar{P}_{\mathrm{s}}= \begin{cases}\mathbb{E}\left\{Q\left(\sqrt{\frac{2|h|^{2} E_{\mathrm{s}}}{\kappa_{\mathrm{I}}(h)+\sigma_{\mathrm{n}}}}\right)\right\} & \text { for BPSK, } \\ 1-\mathbb{E}\left\{\left[1-c Q\left(\sqrt{\frac{a|h|^{2} E_{\mathrm{s}}}{\kappa_{\mathrm{I}}(h)+\sigma_{\mathrm{n}}^{2}}}\right)\right]^{2}\right\} & \text { if } M \geq 4\end{cases}
$$

Above, $Q(\cdot)$ is the Q-function, $c=2(1-1 / \sqrt{M})$ and $a=$ $3 /(M-1)$. The argument of the Q-function is defined as the conditional SINR:

$$
\gamma(h):=\frac{|h|^{2} E_{\mathrm{s}}}{\kappa_{\mathrm{I}}(h)+\sigma_{\mathrm{n}}^{2}},
$$

where the term conditional points out the fact that it depends on the channel realization.

Finally, the mean conditional SINR can be found from (16) as

$$
\bar{\gamma}:=\mathbb{E}\{\gamma(h)\},
$$

being a measure of the power gain of the system, which is relevant to the performance in the low SINR regime [16]. This is further illustrated in Section IV. Note that the mean conditional SINR is a general figure of merit taking into account the interference power correlation. Due to its dependency on the channel, the conditional SINR is a random variable, whose mean value is in general different from the well-known definition of mean SINR, $\mathbb{E}\left\{|h x|^{2}\right\} / \mathbb{E}\left\{|I+n|^{2}\right\}$. These two definitions coincide only when $\kappa_{\mathrm{I}}(h)$ is a constant, i.e. when interference and channel coefficient are uncorrelated. This will be further explored in Section IV.

If the interference follows a distributions that is only approximately Gaussian, then (15) will turn into an approximation. Analyzing the limits up to where this approximation is numerically accurate is beyond the scope of the present work.

\section{B. The Role of the Correlation Sign}

The SINR is a random variable, and as such its simpler characterization involves its mean value and variance. The literature describes effect of an increase of the SINR's mean value over the system performance as "power gains", and effects of a decrease of its variance as "diversity gains" [17]. Examples of the latter are direct to see in scenarios with no interference: the large fluctuations of a Rayleigh channel are related to a poor diversity gain and hence a poor performance, while the zero variance of an AWGN channel corresponds to a maximal diversity gain and hence an exponentially decaying error rate.

Following the results of the previous subsection, a correlation between signal and interference affects the variance of the conditional SINR and hence affects the attainable diversity gain. In effect, when the correlation is positive $\kappa_{\mathrm{I}}(h)$ is an increasing function of $|h|^{2}$, and hence both the numerator and denominator of (16) grow with the channel gain. This reduces the fluctuations of their ratio, providing an increase of the diversity gain with respect to the case of uncorrelated interference - where $\kappa_{\mathrm{I}}(h)$ is a constant. The opposite effect occurs in the case of negative correlation, increasing the variance of the SINR. These larger fluctuations reduce the diversity gain attainable over the channel. 
To further understand the impact of the variance of the SINR on the channel diversity, let us analyze the "Jensen losses" [17] using an approach inspired by the delta method [18]. For simplicity of the mathematical analysis, we focus on a case where the diversity gain of the channel is such that the variance of the conditional SINR, $\sigma_{\gamma}^{2}:=\operatorname{Var}\{\gamma\}$, is small enough so that a second order Taylor approximation around $\bar{\gamma}$ of a given SER function $P_{\mathrm{s}}(\gamma)$ is accurate, i.e.

$$
P_{\mathrm{s}}(\gamma) \approx P_{\mathrm{s}}(\bar{\gamma})+[\gamma-\bar{\gamma}] \frac{d P_{\mathrm{s}}}{d \gamma}(\bar{\gamma})+[\gamma-\bar{\gamma}]^{2} \frac{1}{2} \frac{d^{2} P_{\mathrm{s}}}{d \gamma^{2}}(\bar{\gamma}) .
$$

Then, applying the expected value operator to both sides of (18), one finds that

$$
\bar{P}_{\mathrm{s}} \approx P_{\mathrm{s}}(\bar{\gamma})+\sigma_{\gamma}^{2} \frac{1}{2} \frac{d^{2} P_{\mathrm{s}}}{d \gamma^{2}}(\bar{\gamma}) .
$$

The Jensen's losses refer to the gap between $\bar{P}_{\mathrm{s}}(\bar{\gamma})$ and $P_{\mathrm{s}}(\bar{\gamma})$, as described by Jensen's inequality ${ }^{\S}$. Because of the convexity of $P_{\mathrm{s}}$ (c.f. [19] and references therein), the second term of the right hand side of (19) is positive and approximates the Jensen losses. Moreover, it can be shown that in most cases of interest $d^{2} P_{\mathrm{s}} / d \gamma^{2}$ is an increasing function of $\bar{\gamma}$, and hence the gap created by $\sigma_{\gamma}^{2}$ grows with $\bar{\gamma}$. By noting that the channel diversity is more significant for the high SINR regime [17], it is clear that the Jensen looses are inversely proportional to the diversity gain. Therefore, one can conclude that a large SINR variance induces large Jensen looses, which in turn reduces the diversity gain.

Interestingly, for low values of $\bar{\gamma}$ the second term of (19) is small and hence the Jensen losses are not predominant, resulting in $\bar{P}_{\mathrm{s}} \approx P_{\mathrm{s}}(\bar{\gamma})$. Therefore, in the low SINR regime the power gain associated with $\bar{\gamma}$ is more relevant than the diversity gain (related with $\sigma_{\gamma}^{2}$ ) for the system performance.

\section{PROOF OF CONCEPT}

This section illustrates the findings presented in Section III in a simple scenario. For this, we consider transmissions over narrowband fast-fading channels where the interference power is such that the additive noise can be disregarded, and hence the received signal is $y=h x+I$. The channel coefficient $h$ is assumed to follow a zero-mean circular symmetric complex Gaussian distribution, following the Rayleigh fading model. The same distribution is imposed for $I$ for a given realization of $h$, with variance $\kappa_{\mathrm{I}}(h)$. Three cases are considered:

$$
\kappa_{\mathrm{I}-0}(h)=\sigma_{\mathrm{I}}^{2}, \kappa_{\mathrm{I}-\mathrm{p}}(h)=|h|^{2} \sigma_{\mathrm{I}}^{2}, \kappa_{\mathrm{I}-\mathrm{n}}(h)=\frac{\sigma_{\mathrm{I}}^{2}}{\sqrt{\pi}|h|} .
$$

Case I-0 considers no correlation between the power of the useful signal and the interference, while the positive correlation in I-p was addressed in [7] as a case of "maximally correlated interference", which corresponds to OFDM transmissions as shown in (6) when $u=0$. Finally, I-n considers negative correlation similar to the case of IR-UWB transmissions, as in

\footnotetext{
$\S$ Jensen inequality states that for any convex function $F$ it holds that $\mathbb{E}\{F(X)\} \geq F(\mathbb{E}\{X\})$.
}

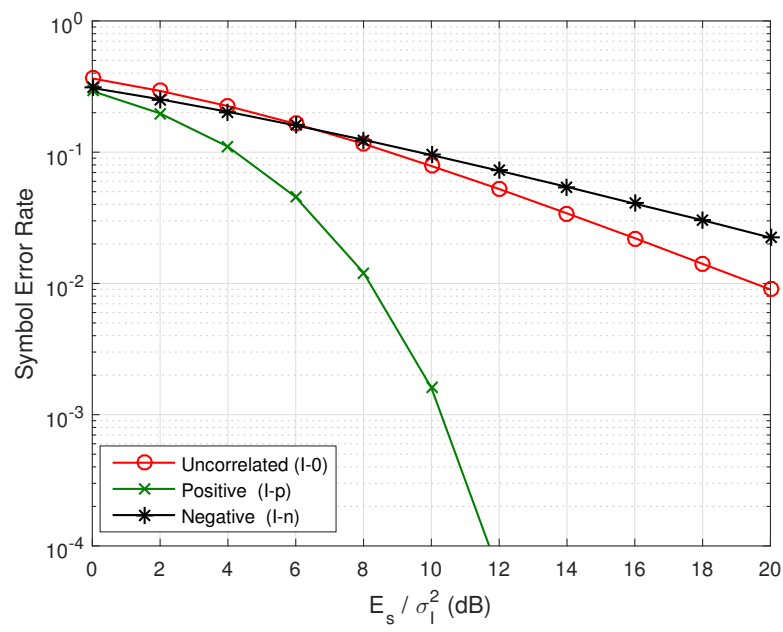

Fig. 1: Error statistics of QPSK transmissions over Rayleigh channels. Note that, beside the having the smaller diversity gain, the negatively correlated case provides a power gain that causes a crossing between its curve and the one from the uncorrelated case.

(12), with a simplified mathematical structure. The term $1 / \sqrt{\pi}$ is a scaling factor so that $\mathbb{E}\left\{\kappa_{\mathrm{I}}(h)\right\}=\sigma_{\mathrm{I}}^{2}$ for all cases.

Applying now the framework presented in Section III-A, the following expressions are found for each scenario:

$$
\gamma_{0}(h)=|h|^{2} \frac{E_{\mathrm{s}}}{\sigma_{\mathrm{I}}^{2}}, \gamma_{\mathrm{p}}(h)=\frac{E_{\mathrm{s}}}{\sigma_{\mathrm{I}}^{2}}, \gamma_{\mathrm{n}}(h)=\sqrt{\pi}|h|^{3} \frac{E_{\mathrm{s}}}{\sigma_{\mathrm{I}}^{2}} .
$$

A direct calculation of their mean value yields

$$
\bar{\gamma}_{0}=\bar{\gamma}_{\mathrm{p}}=\frac{E_{\mathrm{s}}}{\sigma_{\mathrm{I}}^{2}}<\bar{\gamma}_{\mathrm{n}}=3 \pi \frac{E_{\mathrm{s}}}{4 \sigma_{\mathrm{I}}^{2}} .
$$

Also, their variances are

$\operatorname{Var}\left\{\gamma_{\mathrm{p}}\right\}=0<\operatorname{Var}\left\{\gamma_{0}\right\}=\left[\frac{E_{\mathrm{s}}}{\sigma_{\mathrm{I}}^{2}}\right]^{2}<\operatorname{Var}\left\{\gamma_{\mathrm{n}}\right\}=\alpha\left[\frac{E_{\mathrm{s}}}{\sigma_{\mathrm{I}}^{2}}\right]^{2}$

with $\alpha=6 \pi-9 \pi^{2} / 16$.

Simulations of $10^{6}$ random symbols transmitted over independent Rayleigh channels were performed over the three considered scenarios. On the receiver side, per-symbol channel equalization was performed before symbols were demodulated and the error statistics were computed. Results confirmed that the high variance of $\gamma_{\mathrm{n}}$, due to the negative correlation, gives I-n a lower diversity than the standard Rayleigh channel given by I- 0 , as revealed by the slope of the SER curves in the high $E_{\mathrm{s}} / \sigma_{\mathrm{I}}^{2}$ regime (see Figure 1). The larger mean value of $\gamma_{\mathrm{n}}$ gives I-n a power gain, reducing its error rates at low $E_{\mathrm{s}} / \sigma_{\mathrm{I}}^{2}$ values with respect to the uncorrelated case.

Results confirm that the zero variance of $\gamma_{\mathrm{p}}$ generates similar error statistics as for the AWGN channel, thus providing maximal diversity gain. This is a remarkable insight, as the desired signal is still experiencing Rayleigh fading and the only difference with the standard uncorrelated case is the correlation that exists between the interference and useful signal power. 

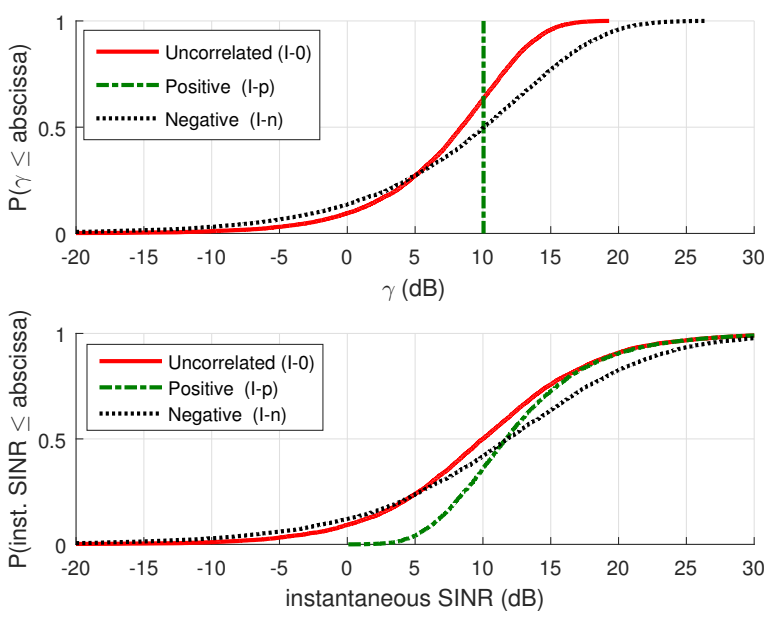

Fig. 2: CDF of the conditional $\operatorname{SINR}(\gamma)$ as given by (21), and of the instantaneous SINR. Both metrics are evaluated for $\sigma_{\mathrm{n}}^{2}=0$ and $E_{\mathrm{s}} / \sigma_{\mathrm{I}}^{2}=10 \mathrm{~dB}$.

Note that the three studied cases have the same ratio of mean signal to interference power, $\mathbb{E}\left\{|h x|^{2}\right\} / \mathbb{E}\left\{|I|^{2}\right\}=E_{\mathrm{s}} / \sigma_{\mathrm{I}}^{2}$. This shows that this metric does not fully reflect the effect of correlated interference over the SER.

Figure 2 illustrates the cumulative distribution function (CDF) of $\gamma$ for all three studied cases, and compares it with the CDF of the instantaneous SINR, which is another well-known metric that in this case corresponds to $|h x|^{2} /|I|^{2}$. It is known that a steeper slope in the CDF of the SINR corresponds to a higher diversity gain, which can be clearly seen in the curves of both the instantaneous and the conditional SINR. However, only the conditional SINR, characterized by a vertical CDF in Figure 2, correctly predicts the AWGN SER of scenario I-p. This finding not only points out the role of the interference correlation on the decoding error statistics, but also clarifies the importance of the conditional SINR as a figure of merit.

\section{CONCLUSIONS}

The conditional SINR, the instantaneous SINR and the ratio between the average useful signal power and the average interference plus noise power are three metrics that extend the traditional SINR. Our results suggest that the conditional SINR is the most accurate performance metric for the mean SER in scenarios where there exist correlations between interference and the desired signal power.

Our framework allows to understand how a positively correlated interference reduces the fluctuations of the conditional SINR, increasing the channel diversity gain. Conversely, a negative correlation reduces the diversity gain, degrading the SER performance. Our methods are applicable to a vast range of communication scenarios, with the only requirement of the interference to approximately follow a Gaussian distribution. We hope that this work can motivate further explorations in scenarios where the knowledge about the effects of the interference's correlation can enable useful insights.

\section{ACKNOWLEDGMENT}

We thank Luca Rugini for helpful discussions. This work was supported by projects FONDECYT 1110370, "Programa de Cooperación Científica Internacional CONICYT/DAAD" and DFG projects CoMP impairments (JU 2793/3-1) and CoMP mobil (JU 2793/4-1). These grants also enabled an enriching exchange with Dr. habil. Volker Jungnickel from the Fraunhofer HHI in Berlin, Germany. Fernando Rosas acknowledges the support of the European Union's H2020 research and innovation programme under the Marie Skłodowska-Curie grant agreement No. 702981. Konstantinos Manolakis thanks Prof. Giuseppe Caire for the fruitful discussions and for supporting this work at the Technische Universität Berlin.

\section{REFERENCES}

[1] N. Bhushan, J. Li, D. Malladi, R. Gilmore, D. Brenner, A. Damnjanovic, R. T. Sukhavasi, C. Patel, and S. Geirhofer, "Network densification: the dominant theme for wireless evolution into $5 \mathrm{~g}$," IEEE Communications Magazine, vol. 52, no. 2, pp. 82-89, February 2014.

[2] H. Boche and M. Schubert, "The structure of general interference functions and applications," Information Theory, IEEE Transactions on, vol. 54, no. 11, pp. 4980-4990, Nov 2008.

[3] M. Haenggi and R. K. Ganti, Interference in large wireless networks. Now Publishers Inc, 2009.

[4] S. Kundu and S. Chakrabarti, "Outage and ber analysis of cellular cdma for integrated services with correlated signal and interference," Communications Letters, IEEE, vol. 7, no. 10, pp. 478-480, Oct 2003.

[5] Z. Xiaodi and N. Beaulieu, "A closed-form ber expression for bpsk using mrc in correlated cci and rayleigh fading," Communications, IEEE Transactions on, vol. 55, no. 12, pp. 2249-2252, Dec 2007.

[6] P. Rahimzadeh and N. Beaulieu, "New simple closed-form ber expressions for mrc diversity bpsk in correlated rayleigh fading and cci," Communications, IEEE Transactions on, vol. 57, no. 3, pp. 630-634, March 2009.

[7] L. Rugini and P. Banelli, "Probability of error of linearly modulated signals with gaussian cochannel interference in maximally correlated rayleigh fading channels," EURASIP Journal on Wireless Communications and Networking, vol. 2010, no. 1, p. 193183, 2010.

[8] L. Halsted, "Coherent echo modulation and detection," Communication Technology, IEEE Transactions on, vol. 14, no. 5, pp. 611-620, October 1966.

[9] M.-S. Alouini and A. Goldsmith, "A unified approach for calculating error rates of linearly modulated signals over generalized fading channels," Communications, IEEE Transactions on, vol. 47, no. 9, pp. 1324-1334, 1999.

[10] H. Sheng and A. M. Haimovich, "Impact of channel estimation on ultrawideband system design," Selected Topics in Signal Processing, IEEE Journal of, vol. 1, no. 3, pp. 498-507, 2007.

[11] A. Koppelaar, S. Drude, M. van Splunter, and A. Hekstra, "On rake processing with estimation errors in a uwb system," in Proceedings of the 36th International Symposium on Information Theory in the Benelux, May 2015, pp. 121-128.

[12] M. Loeve, Probability Theory, 4th ed. Springer, 1978.

[13] C. Oberli, "ML-based tracking algorithms for MIMO-OFDM," IEEE Trans. Wireless Commun., vol. 6, no. 7, pp. 2630-2639, Jul. 2007.

[14] M. García and C. Oberli, "Intercarrier interference in OFDM: a general model for transmissions in mobile environments with imperfect synchronization," EURASIP Journal of Wireless Communications and Networking, 2009, article ID 786040.

[15] A. H. Sayed, Adaptive filters, 1st ed. John Wiley \& Sons, 2008

[16] A. Goldsmith, Wireless Communications. Cambridge University Press, 2005.

[17] D. Tse and P. Viswanath, Fundamentals of Wireless Communication. Cambridge University Press, 2005.

[18] G. Casella and R. L. Berger, Statistical Inference. Duxbury Press, 2002.

[19] A. Rajan and C. Tepedelenlioglu, "A representation for the symbol error rate using completely monotone functions," Information Theory, IEEE Transactions on, vol. 59, no. 6, pp. 3922-3931, June 2013. 